\title{
Model for the Analysis of Membrane-Type Dissolution Tests for Inhaled Drugs
}

\author{
Göran Frenning,* Irès van der Zwaan, Frans Franek, Rebecca Fransson, and Ulrika Tehler
}

Cite This: Mol. Pharmaceutics 2020, 17, 2426-2434

Read Online

ABSTRACT: Impactor-type dose deposition is a common prerequisite for dissolution testing of inhaled medicines, and drug release typically takes place through a membrane. The purpose of this work is to develop a mechanistic model for such combined dissolution and release processes, focusing on a drug that initially is present in solid form. Our starting points are the Noyes-Whitney (or Nernst-Brunner) equation and Fick's law. A detailed mechanistic analysis of the drug release process is provided, and approximate closed-form expressions for the

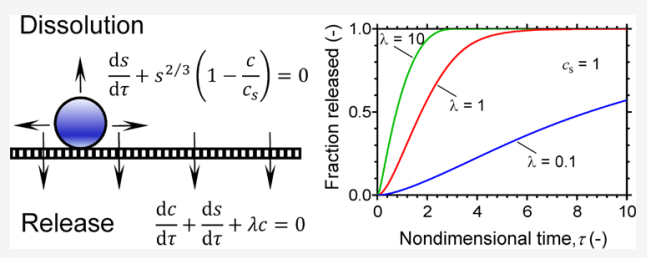
amount of the drug that remains in solid form and the amount of the drug that has been released are derived. Comparisons with numerical data demonstrated the accuracy of the approximate expressions. Comparisons with experimental release data from literature demonstrated that the model can be used to establish rate-controlling release mechanisms. In conclusion, the model constitutes a valuable tool for the analysis of in vitro dissolution data for inhaled drugs.

KEYWORDS: drug delivery, dissolution, lung, mathematical model, formulation development

\section{INTRODUCTION}

In vitro dissolution testing of drug delivery systems is commonly used during the development and manufacturing of dosage forms. Such dissolution tests aid the development and optimization of novel delivery systems and can be used to determine in vitro-in vivo correlations. Moreover, they constitute important quality control tools, but this is currently only utilized for oral drug delivery systems.

Whereas a large number of standardized tests exist for solid dosage forms, no test for orally inhaled products has reached compendial status. However, the development of dissolution tests for delivery systems intended for pulmonary administration has attracted considerable interest, especially during the last decade. ${ }^{1,2}$ Although various design principles have been utilized, adequate dispersion of the drug is a common prerequisite. This is generally accomplished by letting the drug deposit onto a membrane, using an impactor such as the Andersen cascade impactor (ACI) or the next-generation impactor (NGI). The membrane is subsequently transferred to the dissolution equipment, and drug release typically takes place through this membrane.

An early dissolution setup for inhalable powders was based on a flow-through design, similar to the USP Apparatus 4, with a recirculating dissolution medium. ${ }^{3}$ A solid drug was deposited onto a filter that was transferred to a dissolution cell through which the dissolution medium was pumped. Hence, drug transport across the filter was primarily mediated by convection. Likewise, the standard USP Apparatus 1 (rotating basket) has been used to study release from solid lipid microparticles. ${ }^{4}$ Powder samples were wrapped up in sealed glass fiber filters to prevent the microparticles from escaping into the dissolution medium. In this case, drug release is expected to be mediated by a combination of diffusion and convection across the filter.

Many dissolution setups utilize horizontal diffusion cells, as pioneered by Cook et al. ${ }^{5}$ Examples include the modified Franz diffusion cell ${ }^{6,7}$ and diffusion cells based on permeable Transwell supports. ${ }^{8,9}$ Similar designs are utilized for the DissolvIt system ${ }^{10}$ and the method proposed by Eedara et al., ${ }^{11}$ but these setups also include a mucus simulant (and the DissolvIt system is reversed since drug release occurs across a membrane placed on top of the drug). In any case, release across the membrane is expected to be primarily mediated by diffusion.

Also, the standard USP Apparatus 2 (paddle) has been adopted for dissolution studies of inhalable powders. ${ }^{12}$ In this case, drug particles were deposited onto membranes that were mounted in a cassette that was placed in the USP Apparatus 2. Again, drug release is expected to be primarily mediated by diffusion. Recently, a similar setup has been suggested for use in a Pion $\mu$ DISS Profiler. ${ }^{13}$

May et al. ${ }^{14}$ have proposed a model for drug dissolution, based on the Noyes-Whitney/Nernst-Brunner equation, ${ }^{15-17}$ in which consideration was given to the polydispersity of the powders. However, the possible effects of the

Received: February 14, 2020

Revised: May 28, 2020

Accepted: May 28, 2020

Published: May 28, 2020 
membrane that separates the donor from the acceptor compartments were not taken into account.

The purpose of this work is to devise a straightforward mechanistic model that combines drug dissolution, described by the mentioned Noyes-Whitney/Nernst-Brunner equation, ${ }^{15-17}$ and release across a membrane. Hence, the model is formulated for inhalation powders in which the drug initially is present in solid form. A combined dissolution/release model of this type can be used to determine rate-controlling processes and to analyze experimental release data for which the effect of the membrane cannot be disregarded. Although our basic premise is that release is governed by diffusion, as described by Fick's first law, ${ }^{18}$ the proposed model applies also when convection contributes to the release, provided that drug release is proportional to the concentration of the dissolved drug.

\section{MATERIALS AND METHODS}

2.1. Model Formulation. Drug dissolution is assumed to take place in a relatively small donor compartment of volume $V_{\text {liq }}$ that is separated from a considerably larger acceptor compartment by a membrane (Figure 1), both containing the

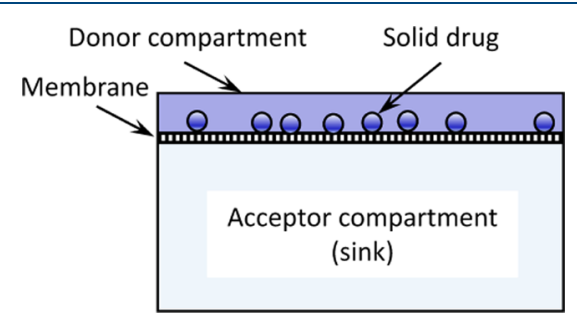

Figure 1. Schematic illustration of the dissolution setup, showing the donor compartment where drug dissolution occurs and the acceptor compartment into which the drug is released by diffusion through the membrane that separates the two compartments.

same type of dissolution medium. This is considered to be a reasonable assumption, although, in reality, some exchange of the dissolution medium may take place between the compartments. The amounts of the solid and dissolved drug in the donor compartment, expressed as mass or moles per unit volume, are specified in terms of variables $S(t)$ and $C(t)$, where $t$ is the time. All drug is assumed to be present in solid form initially. The dissolved drug is assumed to diffuse through the membrane of surface area $A_{\text {mem }}$ and thickness $h_{\text {mem }}$. The effective diffusion coefficient of the drug across the membrane is denoted $D_{\text {mem }}$. Assuming that the acceptor compartment acts as a sink, conservation of mass requires that

$$
V_{\text {liq }}\left(\frac{\mathrm{d} C}{\mathrm{~d} t}+\frac{\mathrm{d} S}{\mathrm{~d} t}\right)=-\frac{A_{\mathrm{mem}} D_{\text {mem }}}{h_{\text {mem }}} C
$$

where the left-hand side represents the rate of change in the total amount of the drug that remains in the donor compartment and the right-hand side is the (negative) amount of the drug that diffuses across the membrane per unit time, as obtained from Fick's first law. ${ }^{18}$ Notice that both $C$ and $S$ depend on time, although the arguments for notational simplicity are not indicated.

With the modifications proposed by Nernst $^{16}$ and Brunner, ${ }^{17}$ the Noyes-Whitney equation ${ }^{15}$ can be expressed as $^{19}$

$$
V_{\text {liq }} \frac{\mathrm{d} S}{\mathrm{~d} t}=-\frac{D_{\text {stag }}}{h_{\text {stag }}} A\left(C_{\mathrm{s}}-C\right)
$$

where $A(t)$ is the total area of the solid drug, $C_{s}$ is the solubility of the drug in the dissolution medium, and $D_{\text {stag }}$ is the diffusion coefficient of the drug across a stagnant layer of thickness $h_{\text {stag. }}$. Assuming fairly monodisperse particles that retain their shape when undergoing dissolution, the relationship between particle surface area and amount of the solid drug can be expressed as $^{20,21}$

$$
\frac{A}{A_{0}}=\left(\frac{S}{S_{0}}\right)^{2 / 3}
$$

where $A_{0}$ and $S_{0}$ denote the initial values of $A$ and $S$, respectively. For simplicity, the thickness of the stagnant layer is here assumed to remain the same throughout the dissolution process, despite the fact that the particles are small. ${ }^{22}$ This assumption is considered satisfactory when the particles are located at the membrane boundary. The amount of the drug that has been released through the membrane up to a certain time $t$ is obtained by integration of the drug release rate, i.e., the magnitude of the right-hand-side of eq 1 . Dividing the result by the total amount of the drug initially present in the system, viz., $M_{0}=V_{\text {liq }} S_{0}$, the fraction of the released drug $u(t)$ is obtained as

$$
u(t)=\frac{A_{\mathrm{mem}} D_{\mathrm{mem}}}{h_{\mathrm{mem}} V_{\mathrm{liq}} S_{0}} \int_{0}^{t} C(x) \mathrm{d} x
$$

where $x$ is a dummy variable.

2.2. Characteristic Time Scale and Nondimensional Form. A characteristic time for diffusion, denoted $t_{\text {diff, }}$ can be defined as follows

$$
t_{\text {diff }}=\frac{h_{\text {mem }} V_{\text {liq }}}{A_{\text {mem }} D_{\text {mem }}}
$$

To see its physical significance, we assume that all drug has dissolved, so that $S=0$. Integration of eq 1 then demonstrates that the concentration of the dissolved drug in the donor compartment decays as $\mathrm{e}^{-t / t_{\text {diff }}}$. In particular, when sink conditions prevail and dissolution is considered to be infinitely fast, the amount of the drug that remains in the donor

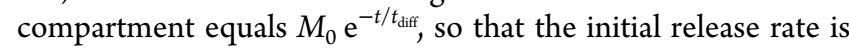
$M_{0} / t_{\text {diff }}$

Similarly, a characteristic time for dissolution, denoted $t_{\text {diss }}$ can be defined as follows

$$
t_{\text {diss }}=\frac{h_{\text {stag }} V_{\text {liq }} S_{0}}{D_{\text {stag }} A_{0} C_{\mathrm{s}}}
$$

To see its physical significance, we note that eq 2 implies that the initial dissolution rate equals $D_{\text {stag }} A_{0} C_{s} / h_{\text {stag }}=M_{0} / t_{\text {diss }}$ since $C=0$ initially, in complete analogy with the result obtained above for $t_{\text {diff }}$.

The ratio between these two time scales will be denoted $\lambda$,i.e.,

$$
\lambda=\frac{t_{\text {diss }}}{t_{\text {diff }}}=\frac{h_{\text {stag }} A_{\text {mem }} D_{\text {mem }} S_{0}}{h_{\text {mem }} A_{0} D_{\text {stag }} C_{\mathrm{s}}}
$$

hence, $\lambda \gg 1$ would correspond to a dissolution-limited release and $\lambda \ll 1$ to a diffusion-limited release, i.e., dissolution is the 
rate-limiting process for $\lambda \gg 1$, whereas diffusion is ratelimiting for $\lambda \ll 1$.

It will be convenient to change the independent variable to $\tau$ $=t / t_{\text {diss }}$. For convenience, we will also introduce the nondimensional variables $c=C / S_{0}$ and $s=S / S_{0}$ together with the nondimensional solubility $c_{\mathrm{s}}=C_{\mathrm{s}} / S_{0}$ (remember that $S_{0}$ is the initial value of $S$ ). Hence, $c_{s}>1$ if all drug can be dissolved in $V_{\text {liq }}$ and $<1$ otherwise. Since it is assumed that all drug exists in solid form initially, $s=1$ and $c=0$ for $\tau=0$. When expressed in nondimensional variables, eq 1 takes the form

$$
\frac{\mathrm{d} c}{\mathrm{~d} \tau}+\frac{\mathrm{d} s}{\mathrm{~d} \tau}+\lambda c=0
$$

Combination of eqs 2 and 3 results in

$$
\frac{\mathrm{d} s}{\mathrm{~d} \tau}+s^{2 / 3}\left(1-\frac{c}{c_{\mathrm{s}}}\right)=0
$$

The fraction of the released drug becomes

$$
u(\tau)=\lambda \int_{0}^{\tau} c(x) \mathrm{d} x
$$

where $x$ is a dummy variable.

2.3. Exact Analysis. The balance equation (eq 8) is linear in the dependent variables and can therefore be readily integrated with respect to $\tau$. One obtains an expression in terms of the fraction of the released drug, cf. eq 10, viz.,

$$
\frac{1 \mathrm{~d} u}{\lambda \mathrm{d} \tau}+u=1-\mathrm{s}
$$

moreover, eq 9 is separable and can be integrated to produce

$$
s^{1 / 3}-1=-\frac{1}{3}\left(\tau-\frac{u}{\lambda c_{s}}\right)
$$

where eq 10 has been used. Combination of eqs 11 and 12 results in a nonlinear first-order ordinary differential equation in terms of $s(\tau)$

$$
\begin{aligned}
3 \frac{\mathrm{d}\left(s^{1 / 3}\right)}{\mathrm{d} \tau} & =s^{-2 / 3} \frac{\mathrm{d} s}{\mathrm{~d} \tau} \\
& =3 \lambda\left(1-s^{1 / 3}\right)+\frac{(1-s)}{c_{\mathrm{s}}}-(1+\lambda \tau)
\end{aligned}
$$

Unfortunately, this equation cannot be solved in closed form. However, as discussed in the following, it forms the basis for an approximate solution procedure. Provided that $s(\tau)$ is known, the fraction of the released drug $u(\tau)$ can be obtained from eq 11 using the method of integrating factor, the result being

$$
u(\tau)=\mathrm{e}^{-\lambda \tau} \int_{0}^{\tau} \lambda \mathrm{e}^{\lambda x}[1-s(x)] \mathrm{d} x=1-\mathrm{e}^{-\lambda \tau}-\lambda H(\tau)
$$

where

$$
H(\tau)=\mathrm{e}^{-\lambda \tau} \int_{0}^{\tau} \mathrm{e}^{\lambda x} s(x) \mathrm{d} x
$$

and $x$ is a dummy variable. The integral in eq 15 changes with time $\tau$ only when the solid drug remains in the donor compartment, i.e., up to a certain time $\tau_{1}$. This implies that $\mathrm{e}^{\lambda \tau} H(\tau)$ and hence $1+\lambda \mathrm{e}^{\lambda \tau} H(\tau)$ are constant for all $\tau>\tau_{1}$. From eq 14 , the constant value attained by this quantity is obtained as $\left(1-u_{1}\right) \mathrm{e}^{\lambda \tau_{1}}$, where $u_{1}=u\left(\tau_{1}\right)$. Using this result in eq 14 , we obtain

$$
u(\tau)=1-\left(1-u_{1}\right) \mathrm{e}^{-\lambda\left(\tau-\tau_{1}\right)}
$$

for $\tau>\tau_{1}$. Alternatively, eq 11 with $s(\tau)=0$ may be integrated, subject to the initial condition $u\left(\tau_{1}\right)=u_{1}$, to produce the same result. In particular, if $c_{\mathrm{s}}>1$ and dissolution would proceed infinitely fast so that $\tau_{1}=u_{1}=0$, the fraction of the released drug would be obtained as $u(\tau)=1-\mathrm{e}^{-\lambda \tau}$. A comparison with eq 14 thus reveals that the function $H(\tau)$ accounts for the retardation of the release caused by a limited dissolution rate or solubility.

If the fraction of the released drug is to be determined numerically, it is more convenient to combine eqs 11 and 12 to obtain the following nonlinear differential equation

$$
\frac{1 \mathrm{~d} u}{\lambda \mathrm{d} \tau}+u=1-\left[1-\frac{1}{3}\left(\tau-\frac{u}{\lambda c_{\mathrm{s}}}\right)\right]^{3}
$$

This equation applies as long as the solid drug remains in the donor compartment, i.e., as long as the quantity within the square brackets is positive. It corresponds to eq 8 in the work by Frenning et al., ${ }^{23}$ where a similar procedure was used.

2.4. Asymptotic Behaviors. It is instructive to study the behavior of $s(\tau)$ when $\lambda \gg 1$ and $\lambda \ll 1$, i.e., the asymptotic behaviors of $s(\tau)$ when $\lambda \rightarrow \infty$ and $\lambda \rightarrow 0$.

2.4.1. Dissolution-Controlled Release. When the characteristic time for dissolution is significantly larger than that for diffusion (i.e., when $\lambda \gg 1$ ), accumulation of the dissolved drug in the donor compartment can be neglected. In this case, eq 9 reduces to a separable ordinary differential equation in $s(\tau)$, with solution

$$
s^{1 / 3}-1=-\frac{\tau}{3}
$$

which is the well-known Hixson-Crowell cube-root law. ${ }^{20}$ Clearly, this result is immediately obtained from eq 12 in the limit $\lambda \rightarrow \infty$. Hence, the fraction of the drug that remains in solid form can be expressed as

$$
s=\left(1-\frac{\tau}{3}\right)^{3}
$$

Notice that drug dissolution according to eqs 18 or 19 depends solely on the characteristic time for dissolution since $\tau=t / t_{\text {diss }}$, which is expected when $\lambda \gg 1$.

2.4.2. Diffusion-Controlled Release. When the characteristic time for dissolution is significantly smaller than that for diffusion across the membrane (i.e., when $\lambda \ll 1$ ), drug release will be biphasic. First, a rapid initial drug dissolution will occur, until either a saturated solution is obtained in the donor compartment or all drug has dissolved, depending on the initial amount of the solid drug present. When the initial drug loading exceeds the solubility (i.e., when $c_{\mathrm{s}}<1$ ), a steady state with $c=$ $c_{s}$ will thereafter be maintained as long as the solid drug remains in the donor compartment. Putting $\mathrm{d} c / \mathrm{d} \tau=0$ and $c=$ $c_{\mathrm{s}}$ in eq 8 , one finds that

$$
\frac{\mathrm{d} s}{\mathrm{~d} \tau}=-\lambda c_{\mathrm{s}}
$$

implying that

$$
s=\left(1-c_{\mathrm{s}}\right)-c_{\mathrm{s}} \lambda \tau
$$


where $\left(1-c_{s}\right)$ represents the drug that remains in solid form after the initial (essentially instantaneous) drug dissolution. The above expression is valid as long as the solid drug remains. Thereafter, eq 16 applies. Notice that the fraction of the drug that remains in solid form according to eq 21 depends on time through the product $\lambda \tau=\left(t_{\text {diss }} / t_{\text {diff }}\right)\left(t / t_{\text {diss }}\right)=t / t_{\text {diff, }}$ i.e., only on the characteristic time for diffusion, as expected when $\lambda \ll 1$.

2.4.3. Sink Conditions. Sink conditions prevail whenever the solubility significantly exceeds the initial drug loading, i.e., when $c_{\mathrm{s}} \gg 1$. In this case, eqs 18 and 19 continue to be valid, irrespective of the value of $\lambda$.

2.5. Approximate Analysis. To obtain an approximate solution of the drug release problem, we first seek an expression for the rapid initial drug dissolution obtained when $\lambda \ll 1$ and $c_{\mathrm{s}}<1$. To this end, we change variable to $w=$ $1-s$ in eq 13 . Since $w \ll 1$ initially, we keep only the linear terms to obtain

$$
\frac{\mathrm{d} w}{\mathrm{~d} \tau}+(\mu+\nu \tau) w=1+\lambda \tau
$$

where $\mu=2 / 3+\lambda+1 / c_{\mathrm{s}}$ and $\nu=2 \lambda / 3$. The solution of eq 22 may be expressed in terms of Airy functions ${ }^{24}$ but is cumbersome in the subsequent developments. Consistent with the fact that $\lambda \ll 1$ and that we are interested in the initial drug dissolution, we therefore neglect $\nu \tau$ in comparison with $\mu$. For the same reason, we neglect $\lambda \tau$ in comparison with 1 on the right-hand side. With these simplifications, the solution of eq 22 subject to the initial condition $w(0)=0$ takes the form

$$
w=1-s=\frac{1-\mathrm{e}^{-\mu \tau}}{\mu}
$$

Consistent with the limiting results expressed by eqs 19 and 21 and the expression for the initial drug dissolution embodied in eq 23 , we postulate the following approximate form for $s$

$$
\tilde{s}=\frac{\left(A \mathrm{e}^{-\mu \tau}+B\right)\left(1-\frac{\tau}{\tau_{1}}\right)^{3}}{\left(1-\frac{a \tau}{\tau_{1}}\right)^{2}}
$$

Provided that $\tau_{1}$ is known, the constants $A, B$, and $a$ can be determined fairly conveniently from the conditions that the functional value and the first two derivatives of $\tilde{s}$ attain the correct values at $\tau=0$. The derivations are provided in Appendix A. It turns out to be more involved to determine an accurate value for $\tau_{1}$ itself, which represents the value of $\tau$ at which dissolution is complete. Our approach is based on demanding that eq 13 be satisfied when $\tau=\tau_{1}$. This condition can be translated to a nonlinear algebraic equation from which $\tau_{1}$ can be determined. Again, the derivations are summarized in Appendix A.

When evaluating the integral that results when $\tilde{s}$ is substituted for $s$ in eq 15 , we proceed slightly differently depending on the value of the constant $a$. When $a \neq 0$, we change variable to $\sigma=1-a \tau / \tau_{1}$ and let $b=1-a$, so that

$$
\begin{aligned}
H(\tau)= & -\frac{\tau_{1}}{a^{4}} \mathrm{e}^{-\lambda \tau} \\
& \int_{1}^{\sigma} \frac{\left[A \mathrm{e}^{\left(\lambda_{1}-\mu_{1}\right)(1-x)}+B \mathrm{e}^{\lambda_{1}(1-x)}\right](x-b)^{3}}{x^{2}} \mathrm{~d} x
\end{aligned}
$$

where $x$ is a dummy variable. For convenience, we have used the shorthand notation $\lambda_{1}=\lambda \tau_{1} / a$ and $\mu_{1}=\mu \tau_{1} / a$. It proves convenient to introduce the auxiliary function

$$
\begin{aligned}
F(\alpha, b ; x) \stackrel{\text { def }}{=} & -\mathrm{e}^{-\alpha(1-x)} \int \frac{\mathrm{e}^{\alpha(1-x)}(x-b)^{3}}{x^{2}} \mathrm{~d} x \\
= & {\left[\left(\frac{1}{\alpha^{2}}-\frac{3 b-x}{\alpha}-\frac{b^{3}}{x}\right)\right.} \\
& \left.-\left(3 b^{2}+\alpha b^{3}\right) f(\alpha x)\right]
\end{aligned}
$$

where the constant of integration has been omitted and where $f(x)$ is a function defined as

$$
f(x) \stackrel{\text { def }}{=} \mathrm{e}^{x} \operatorname{Ei}(-x)
$$

with Ei being an exponential integral. ${ }^{24}$ Moreover, to evaluate the integral in eq 25, partial fraction decomposition of the rational function in $x$ and integration by parts have been performed. Using eq 26 in eq 25 , we obtain

$$
\begin{aligned}
H(\tau)= & \frac{\tau_{1}}{a^{4}}\left[A F\left(\lambda_{1}-\mu_{1}, b ; \sigma\right) \mathrm{e}^{-\mu \tau}+B F\left(\lambda_{1}, b ; \sigma\right)\right. \\
& \left.-K_{1} \mathrm{e}^{-\lambda \tau}\right]
\end{aligned}
$$

where $K_{1}=A F\left(\lambda_{1}-\mu_{1}, b ; 1\right)+B F\left(\lambda_{1}, b ; 1\right)$ is an auxiliary constant that represents the contribution from the lower endpoint of the integral in eq 25 . Visual basic routines that can be used to determine the functions $f(x), F(\alpha, b ; x)$ and $H(\tau)$ using, e.g., Microsoft Excel are provided in the Supporting Information.

The above analysis cannot be used when $a=0$ (or very small) since $a$ appears in denominators. Such $a$ values are to be expected when sink conditions prevail since $s(\tau)$ then approaches the asymptotic form given by eq 19 , which is identical in form to $\tilde{s}$ obtained from eq 24 when $a=A=0$. In the special case of $a=0$ (or very small), we instead change variable to $\rho=1-\tau / \tau_{1}$ to obtain

$$
H(\tau)=-\tau_{1} \mathrm{e}^{-\lambda \tau} \int_{1}^{\rho}\left[A \mathrm{e}^{\left(\lambda_{2}-\mu_{2}\right)(1-x)}+B \mathrm{e}^{\lambda_{2}(1-x)}\right] x^{3} \mathrm{~d} x
$$

where $\lambda_{2}=\lambda \tau_{1}$ and $\mu_{2}=\mu \tau_{1}$ and $x$ again is a dummy variable. Defining the auxiliary function

$$
\begin{aligned}
G(\alpha ; x) & \stackrel{\text { def }}{=}-\mathrm{e}^{-\alpha(1-x)} \int \mathrm{e}^{\alpha(1-x)} x^{3} \mathrm{~d} x \\
& =\frac{(\alpha x)^{3}+3(\alpha x)^{2}+6 \alpha x+6}{\alpha^{4}}
\end{aligned}
$$

where the constant of integration has been omitted, we may in analogy with eq 28 write

$$
H(\tau)=\tau_{1}\left[A G\left(\lambda_{2}-\mu_{2} ; \rho\right) \mathrm{e}^{-\mu \tau}+B G\left(\lambda_{2} ; \rho\right)-K_{2} \mathrm{e}^{-\lambda \tau}\right]
$$

where $K_{2}=A G\left(\lambda_{2}-\mu_{2} ; 1\right)+B G\left(\lambda_{2} ; 1\right)$ is an auxiliary constant that represents the contribution from the lower endpoint of the integral in eq 29. The fraction of the released drug is finally obtained using $H(\tau)$ as obtained from eq 28 or 31 in eq 14 . Visual basic routines that can be used to determine the functions $G(\alpha ; x)$ and $H(\tau)$ using Excel are provided in the Supporting Information. 

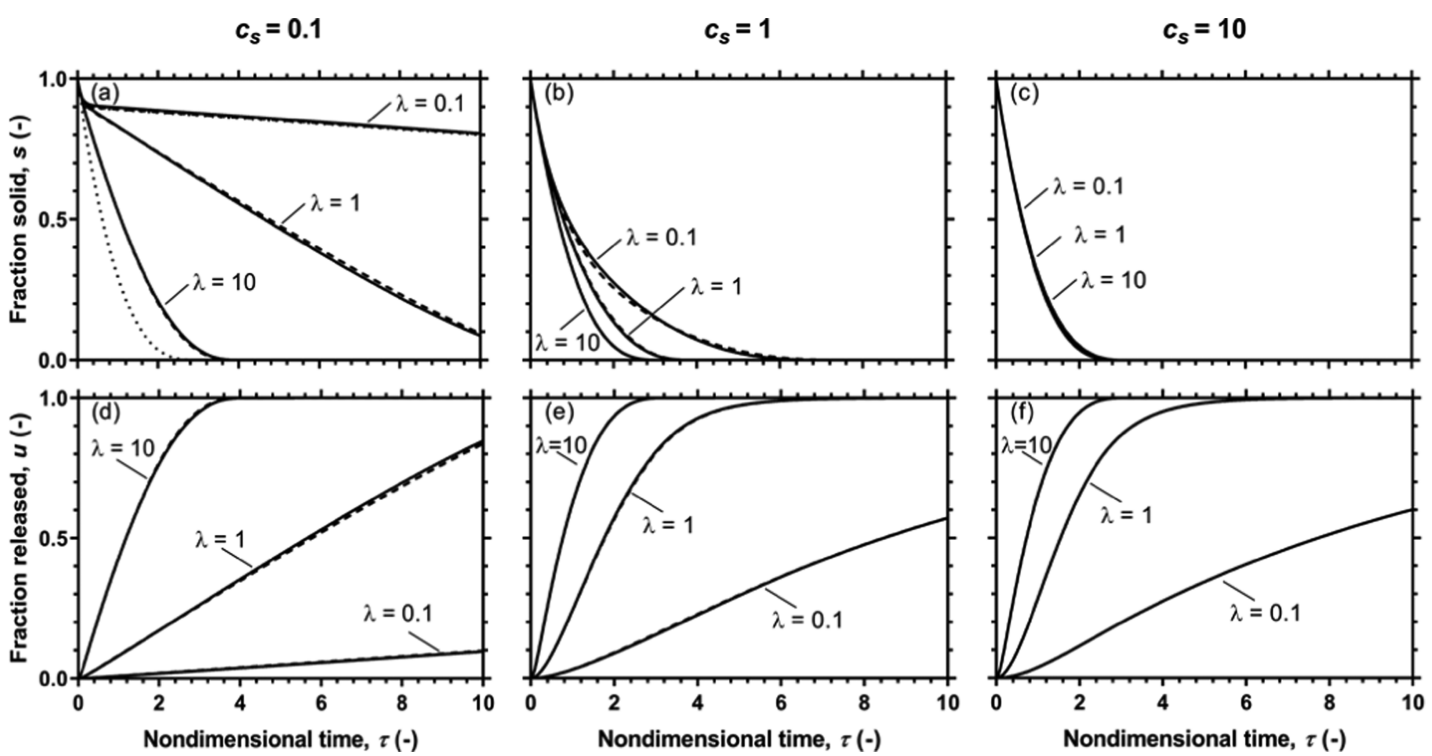

Figure 2. Fraction of solid drug $s$ remaining in the donor compartment $(\mathrm{a}-\mathrm{c})$ and the fraction of the released drug $u(\mathrm{~d}-\mathrm{f})$ vs nondimensional time $\tau$ as obtained analytically (solid lines) and numerically (dashed lines). Model calculations were performed for $c_{\mathrm{s}}=0.1(\mathrm{a}, \mathrm{d}), c_{\mathrm{s}}=1(\mathrm{~b}, \mathrm{e})$, and $c_{\mathrm{s}}=$ 10 (c, f). In each case, results are presented for $\lambda=0.1,1$ and 10 . For comparison, the limiting results obtained when $\lambda \ll 1$ and $\lambda \gg 1$ are included in the top-left graph (dotted lines).

2.6. Numerical Evaluation. The standard NewtonRaphson method, with an initial value of $1 / 3$, was used to solve eq A16 for $\omega=1 / \tau_{1}$ (cf. Appendix A and Supporting Information). The function $H(\tau)$ was evaluated by eq 28 when $a>0.01$ and by eq 31 otherwise. The exponential integral was evaluated using an Excel VBA implementation of the algorithm described by Paciorek (see the Supporting Information). ${ }^{25}$ The approximate analytical solutions were compared to numerical solutions obtained using the Runge-Kutta Fehlberg method implemented in Maple 2019.1 (Maplesoft, Canada).

\section{RESULTS AND DISCUSSION}

3.1. Assessment of Accuracy and Parametric Study. Model calculations were performed for three different values of the nondimensional solubility $c_{\mathrm{s}}(0.1,1$, and 10$)$, defined as the ratio between the solubility of the drug in the donor compartment and the initial drug loading. For each value of $c_{s}$, three different values of the ratio $\lambda(0.1,1$, and 10$)$ between the characteristic times for dissolution and diffusion were considered. The results obtained are summarized in Figure 2. Specifically, Figure $2 \mathrm{a}-\mathrm{c}$ displays the fraction of solid drug $s$ remaining in the donor compartment, and Figure $2 \mathrm{~d}-\mathrm{f}$ shows the fraction of drug $u$ that has been released to the acceptor compartment. The results obtained for $c_{s}=0.1$ are shown in Figure $2 \mathrm{a}, \mathrm{d}$, for $c_{\mathrm{s}}=1$ in Figure $2 \mathrm{~b}, \mathrm{e}$, and for $c_{\mathrm{s}}=10$ in Figure $2 c, f$.

Before discussing the individual cases in detail, we note that the analytical approximation (solid lines) generally exhibits a good to excellent correspondence with the numerical results (dashed lines). Minor deviations can be seen especially when diffusion across the membrane has a significant impact on the dissolution and release profiles. This is expected because drug dissolution and release by diffusion become more interrelated in this case. However, also, in this case, an adequate correspondence is observed. The analytical approximation has been tested in a wider parameter range, and its correspondence to the analytical solution remains adequate. For example, the maximal absolute errors observed in the parameter range $0.01 \leq c_{s}, \lambda \leq 100$ were 0.033 for $s$ and 0.022 for $u$. Hence, we can conclude that the analytically derived expressions can be used with confidence, e.g., in the analysis of experimental release data (cf. Section 3.2 below).

Figure $2 \mathrm{a}, \mathrm{d}$ corresponds to dissolution and release when no more than $10 \%$ of the initial drug loading can be dissolved in the donor compartment. Depending on the value of $\lambda$, drug release ranges from being largely dissolution-controlled (for $\lambda$ $=10$ ) to being largely diffusion-controlled (for $\lambda=0.1$ ). This is corroborated by a comparison with the limiting results obtained for $\lambda \ll 1$ and $\lambda \gg 1$, embodied in eqs 19 and 21 , which are included in Figure 2a (dotted lines). It can be noted that a value of $\lambda>10$ is needed to obtain a complete dissolution control since the solubility is low. On the contrary, the agreement with the diffusion-controlled result is almost perfect when $\lambda=0.1$. In this case, a rapid initial drug dissolution is clearly seen in the fraction of the solid drug remaining in the donor compartment, cf. eq 23, which corresponds to a small delay in the fraction of the drug being released. Since only a small fraction of the total amount of the drug can be dissolved in the donor compartment, the fraction of the released drug closely mirrors the amount of the drug being dissolved.

Figure $2 \mathrm{~b}$,e corresponds to dissolution and release when all of the initial drug loading (but no more) can be dissolved in the donor compartment. Drug release is dissolution-controlled for $\lambda=10$, and the limited solubility in the donor compartment clearly affects dissolution for smaller values of $\lambda$. However, this effect is not as pronounced as it was for $c_{s}=0.1$, as expected.

Figure $2 c$,f corresponds to dissolution and release when a dose 10 times larger than the one present can be dissolved in the donor compartment. Since dissolution occurs under sink conditions, the results obtained for the fraction of the solid drug remaining in the donor compartment collapse on a single curve, corresponding to the limiting result expressed by eq 19 . The parameter $\lambda$ nevertheless has a decisive influence on the fraction of the released drug since it controls the rate at which the dissolved drug diffuses across the membrane. 
3.2. Comparison with Experimental Release Data. When expressed in the nondimensional form, the fraction of the released drug depends on drug solubility in relation to the initial drug loading $\left(c_{\mathrm{s}}=\mathrm{C}_{\mathrm{s}} / S_{0}\right)$ and the ratio between the time scales for dissolution and diffusion $\left(\lambda=t_{\text {diss }} / t_{\text {diff }}\right)$. For real release data, the time scale for dissolution $\left(t_{\text {diss }}\right)$ is needed to convert dimensional time to nondimensional time according to $\tau=t / t_{\text {diss }}$. Hence, the fractional release $u(t)$ generally depends on three parameters, which, e.g., may be selected as $t_{\text {diss }}, t_{\text {diff }}$ and $c_{s}=C_{s} / S_{0}$. The situation is different when sink conditions prevail, however, since these parameters are dependent. The reason for this is that dissolution no longer is impeded by a limited solubility, implying that $u(t)$ becomes independent of $c_{s}$. The dissolution profile is well described by the asymptotic equation (eq 19) whenever $c_{s} \gg 1$, which is identical in form to the profile described by eq 24 if $a=A=0$ (and consequently $B=1$ ) and $\tau_{1}=3$. Hence, eq 31 reduces to

$$
H=3\left[G\left(3 \lambda ; 1-\frac{\tau}{3}\right)-G(3 \lambda ; 1) \mathrm{e}^{-\lambda \tau}\right]
$$

so that, according to eq 14

$$
u(\tau)=1-\mathrm{e}^{-\lambda \tau}-3 \lambda\left[G\left(3 \lambda ; 1-\frac{\tau}{3}\right)-G(3 \lambda ; 1) \mathrm{e}^{-\lambda \tau}\right]
$$

As seen from the above equation, the fraction of the released drug thus depends on time via the two quantities $\tau=t / t_{\text {diss }}$ and $\lambda \tau=t / t_{\text {diff. }}$. Hence, $t_{\text {diss }}$ and $t_{\text {diff }}$ between them define the release profile and no value for $c_{\mathrm{s}}$ needs to be provided.

Moreover, an incomplete release is often seen in experimental release data, i.e., the fraction of the released drug levels out at a value smaller than $100 \%$. This may be caused by a loss of the drug during deposition or binding of the drug to surfaces or materials during the release experiment. To accommodate for this phenomenon in a simplified manner, the theoretical amount of the released drug can be multiplied by a constant $N<1$.

3.2.1. Data from Sakagami et al. Sakagami et al. have recently presented dissolution data for a range of orally inhaled corticosteroid products using a Transwell-based dissolution setup. $^{26}$ The dissolution medium consisted of $10 \mathrm{~mL}$ of simulated lung-lining fluid with $0.02 \% \mathrm{w} / \mathrm{v}$ dipalmitoyl phosphatidylcholine (DPPC) in the donor compartment. For the more soluble corticosteroids flunisolide (FN), triamcinolone acetonide (TA), and budesonide (BUD), the same dissolution medium was used in the acceptor compartment. However, for the less soluble ones, $1 \% \mathrm{w} / \mathrm{v}$ D- $\alpha$-tocopheryl poly(ethylene glycol) 1000 succinate (TPGS) was added to the dissolution medium in the acceptor compartment. The addition of TPGS to only one compartment makes the interpretation of the results more difficult since some movement of the dissolution medium between the compartments likely occurs. For this reason, we here focus on the more soluble steroids, for which the dissolution profiles presented in Figure 3 were obtained. ${ }^{26}$ The parameter $c_{\mathrm{s}}$ was calculated from data obtained from Sakagami et al. ${ }^{26}$ and $N$ was set equal to 1 since a complete dissolution was observed for all three drugs (Table 1). The characteristic time for diffusion across the membrane $\left(t_{\text {diff }}\right)$ was determined from data for FN (dotted curve), for which dissolution was found to be very rapid. ${ }^{26}$ Varying the characteristic times for dissolution $\left(t_{\text {diss }}\right)$ while keeping $t_{\text {diff }}$ fixed at the value obtained for FN resulted in the

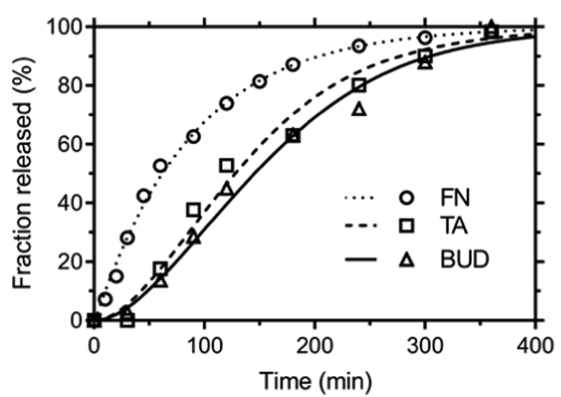

Figure 3. Comparison between experimental data (symbols) from Sakagami et al. ${ }^{26}$ and model calculations (dotted, dashed, and solid lines) using the parameters collected in Table 1 for drugs flunisolide (FN), triamcinolone acetonide (TA), and budesonide (BUD).

Table 1. Parameters Used in the Model Calculations Presented in Figure 3 for Drugs Flunisolide (FN), Triamcinolone Acetonide (TA), and Budesonide (BUD)

$\begin{array}{ccccc}\text { substance } & c_{\mathrm{s}}(-) & t_{\text {diss }}(\min ) & t_{\text {diff }}(\min ) & N(-) \\ \text { FN } & 17.9^{a} & \ll 1 & 88.0 & 1 \\ \text { TA } & 4.64^{a} & 75.5 & 88.0^{b} & 1 \\ \text { BUD } & 4.29^{a} & 97.0 & 88.0^{b} & 1\end{array}$

${ }^{a}$ Calculated from data obtained from Sakagami et al. ${ }^{26}$ Kept constant at the value obtained for FN.

dashed and solid lines corresponding to TA and BUD, respectively. For numerical reasons, $t_{\text {diss }}$ was required to be at least $10^{-3} \mathrm{~min}$ (indicated as $\ll 1 \mathrm{~min}$ in Table 1 ). The agreement between the theoretical release profiles and the experimental data is considered satisfactory, especially since notable variations between repeated experiments were observed by Sakagami et al. ${ }^{26}$ These results indicate that the difference seen between the drugs indeed can be attributed to differences in their solubility.

3.2.2. Data from Eedara et al. Eedara et al. have recently described a novel dissolution method for inhaled drugs that consists of a donor compartment that is separated from a flowthrough cell by a dialysis membrane. ${ }^{11}$ The method was used to investigate dissolution and diffusional transport of the antitubercular drugs moxifloxacin and ethionamide using phosphate-buffered saline (PBS) as the dissolution medium. Poly(ethylene oxide) (PEO) was used to simulate the mucus layer. The results obtained for ethionamide exhibited a release profile that resembled the classical Higuchi square-root-of-time law, ${ }^{27,28}$ indicating that other mechanisms than those considered in this work may be at work. Examples of release profiles obtained for moxifloxacin at different perfusion rates, as obtained by Eedara et al., ${ }^{11}$ are presented in Figure 4. Some data at larger times have been excluded to more clearly display the initial delay. Moreover, the release profiles exhibited a gradual convergence toward complete release for larger times (data not shown), which here is interpreted as resulting from a slow release of the drug embedded in the PEO matrix. For this reason, we focus on the initial release and put $N$ equal to 0.9 . The parameter $c_{\mathrm{s}}$ (Table 2) was calculated from data provided by Eedara et al. ${ }^{11}$ The release profiles in Figure 4 exhibit a dependence on the permeation rate and seem to converge for sufficiently high rates. As noted by the authors, this behavior is consistent with the one expected from the presence of an unstirred water layer, which effectively acts as external masstransfer resistance. ${ }^{29,30}$ For this reason, the characteristic time for dissolution $\left(t_{\text {diss }}\right)$ was determined from data for a perfusion 


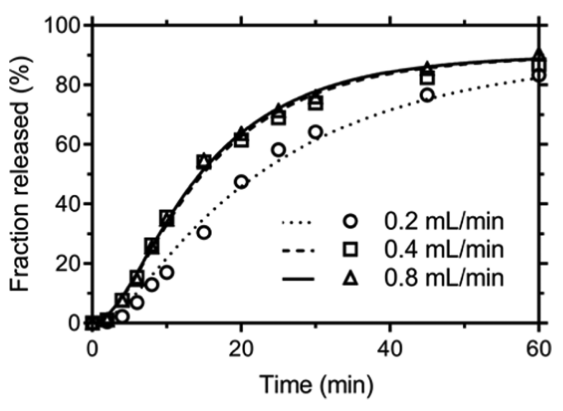

Figure 4. Comparison between experimental data (symbols) from Eedara et al. ${ }^{11}$ and model calculations (lines) using the parameters collected in Table 2.

Table 2. Parameters Used in the Model Calculations Presented in Figure 4

$\begin{array}{ccccc}\text { permeation rate }(\mathrm{mL} / \mathrm{min}) & c_{s}(-) & t_{\text {diss }}(\min ) & t_{\text {diff }}(\min ) & N(-) \\ 0.2 & 8.84^{a} & 4.52^{b} & 22.9 & 0.9 \\ 0.4 & 8.84^{a} & 4.52^{b} & 13.5 & 0.9 \\ 0.8 & 8.84^{a} & 4.52 & 13.1 & 0.9\end{array}$

${ }^{a}$ Calculated from data obtained from Eedara et al. ${ }^{11}{ }^{b}$ Kept constant at the value obtained for a permeation rate of $0.8 \mathrm{~mL} / \mathrm{min}$.

rate of $0.8 \mathrm{~mL} / \mathrm{min}$ (solid curve in Figure 4). Varying the characteristic times for diffusion $\left(t_{\text {diff }}\right)$ while keeping $t_{\text {diss }}$ fixed at the value obtained for $0.8 \mathrm{~mL} / \mathrm{min}$ resulted in the dotted and dashed lines corresponding to the perfusion rates of 0.2 and $0.4 \mathrm{~mL} / \mathrm{min}$, respectively. Again, the correspondence between theory and experiments is considered satisfactory, especially since there are inherent uncertainties in the experimental data, corroborating the interpretation that the differences seen between perfusion rates are due to an external mass-transfer resistance (unstirred water layer).

3.2.3. Data from Rohrschneider et al. Rohrschneider et al. have presented dissolution and release data for the three inhaled corticosteroids BUD, ciclesonide (CIC), and fluticasone propionate (FP) using a Transwell-based dissolution setup. ${ }^{31}$ To reduce the diffusional resistance, the polycarbonate membrane was removed and the glass microfiber filter/filter paper onto which the drug was deposited was placed on thermoformed notches in the Transwell insert. Dissolution experiments were generally performed using $0.5 \% \mathrm{w} / \mathrm{v}$ sodium dodecyl sulfate (SDS) in the PBS solution. Examples of the results obtained are shown in Figure $5 .^{31}$ The parameter $c_{\mathrm{s}}$ was calculated from data obtained from Rohrschneider et al. ${ }^{31}$ The parameter $N$ was set to 1 for BUD and CIC since a nearly complete release occurred for these drugs. The remaining parameters were determined by curve fitting, see Table 3 .

Table 3. Parameters Used in the Model Calculations Presented in Figure 5 for Drugs Budesonide (BUD), Ciclesonide (CIC), and Fluticasone Propionate (FP)

\begin{tabular}{|ccccc}
\hline substance & $c_{\mathrm{s}}(-)$ & $t_{\text {diss }}(\min )$ & $t_{\text {diff }}(\min )$ & $N(-)$ \\
\hline BUD & $2.35^{a}$ & 1.26 & 33.9 & 1 \\
CIC & $2.00^{a}$ & $\ll 1$ & 66.8 & 1 \\
FP & $0.20^{a}$ & 158 & $\ll 1$ & 0.65
\end{tabular}

${ }^{a}$ Calculated from data obtained from Rohrschneider et al. ${ }^{31}$

Hence, the characteristic times for dissolution and diffusion $\left(t_{\text {diss }}\right.$ and $\left.t_{\text {diff }}\right)$ were both allowed to vary. However, for numerical reasons, both characteristic times were required to be at least $10^{-3} \mathrm{~min}$ (indicated as $\ll 1 \mathrm{~min}$ in Table 3 ). The results are shown by solid lines in Figure 5.

The model results suggest that release is diffusion-controlled for both BUD and CIC for which $c_{\mathrm{s}}>1$ so that all drug can be dissolved in the donor compartment. This result may appear somewhat surprising since a clear difference between release of the drug and release of the solution was observed by Rohrschneider et al. ${ }^{31}$ but can be explained by differences in $\lambda$, i.e., the ratio between the characteristic time scales for dissolution and diffusion. That diffusion dominates can also be seen in Figure $5 b$, which displays the fraction of the remaining drug (i.e., $1-u)$ on a logarithmic scale. If all drug dissolves rapidly in the donor compartment, the fraction of the remaining drug is expected to decrease $\mathrm{e}^{-t_{\text {diff }} / t}$ [see the discussion following eqs 5 and 16 with $\left.u_{1}=\tau_{1}=0\right]$, so that a linear decrease would be seen in a semilog plot. This is indeed found, especially for CIC but also for BUD (notice that deviations from a linear relationship may occur at low fractions of the remaining drug because less than $100 \%$ of the drug may be released). It would be tempting to attribute the differences seen in the release rate between BUD and CIC to differences in solubility. However, provided that $c_{\mathrm{s}}>1$ and dissolution is rapid, other factors are likely involved since the initial release rate then equals $M_{0} / t_{\text {diff, }}$ where $M_{0}$ is the total amount of the drug present in the system and $t_{\text {diff }}$ only depends on the diffusion coefficient across the membrane and geometrical factors [eq 5 and the discussion following that equation]. Although a higher solubility does result in a higher release rate in terms of grams or moles per second, a larger amount of the drug needs to be transported, so that the fractional release rate will be independent of solubility.
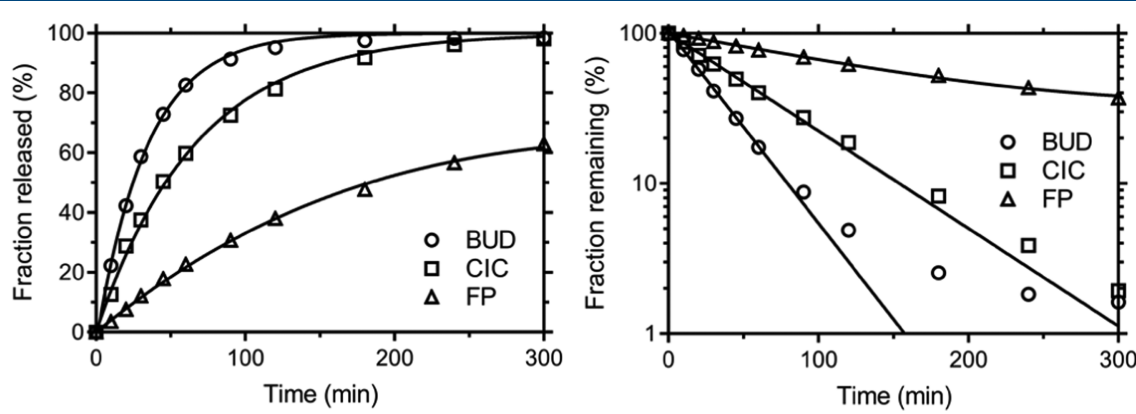

Figure 5. Comparison between experimental data (symbols) from Rohrschneider et al. ${ }^{31}$ and model calculations (solid lines) using the parameters collected in Table 3 for drugs budesonide (BUD), ciclesonide (CIC), and fluticasone propionate (FP). Fraction of the released drug (left) and a semilog plot of the fraction of the remaining drug (right). 
The situation is different for FP, where our data suggests that the release is completely dissolution-controlled and that only about $65 \%$ of the drug is released. An incomplete release of FP is consistent with other data presented by Rohrschneider et al. ${ }^{31}$ and may, for example, be the result of losses of the drug due to its binding to surfaces in the dissolution setup.

\section{CONCLUSIONS}

A model of dissolution based on the well-established NoyesWhitney/Nernst-Brunner equation coupled with diffusional transport across a membrane was formulated and expressed in nondimensional form. A closed-form analytical approximation was derived. This approximation has sufficient accuracy to be used with confidence irrespective of the rate-controlling mechanism(s). The usefulness of the model to establish ratecontrolling mechanisms was demonstrated by comparisons with experimental release data obtained from the literature.

\section{APPENDIX A}

At $\tau=0$, expression 24 reduces to

$$
\tilde{s}(0)=A+B=1
$$

where the second equality follows from the initial condition for $s$. By straightforward differentiation of $\tilde{s}$, one obtains

$$
\tilde{s}^{\prime}(0)=-\mu A-\frac{3-2 a}{\tau_{1}}
$$

and

$$
\tilde{s}^{\prime \prime}(0)=\mu^{2} A+\frac{2 \mu(3-2 a) A}{\tau_{1}}+\frac{6(1-a)^{2}}{\tau_{1}^{2}}
$$

where the primes denote differentiation with respect to $\tau$ and where use has been made of eq A1. Inserting the initial values $\tau$ $=0$ and $s=1$ in eq 13 , it is seen that

$$
s^{\prime}(0)=-1
$$

Similarly, differentiating eq 13 with respect to $\tau$ and inserting the initial values stated above, one finds that

$$
s^{\prime \prime}(0)=\frac{2}{3}+\frac{1}{c_{s}}
$$

The above equations simplify somewhat upon letting $a=1-b$ (as in the main text). Also, introducing $\beta=b / \tau_{1}$ and $\omega=1 / \tau_{1}$, we find by a combination of eqs $\mathrm{A} 2$ and $\mathrm{A} 4$ that

$$
\mu A+2 \beta+\omega=1
$$

Similarly, using the above substitutions in eq A3, we obtain

$$
\mu A(\mu+4 \beta+2 \omega)+6 \beta^{2}=s^{\prime \prime}(0)
$$

where $s^{\prime \prime}(0)$ is used as a shorthand notation for the value provided by eq A5. Solving eq A6 for $\mu A$ and substituting the result in eq $A 7$, we obtain an equation of the form

$$
\beta^{2}+4 \phi \beta+\phi^{2}=\varepsilon
$$

where

$$
\phi=\omega+\frac{\mu}{4}-\frac{1}{2}
$$

and

$$
\varepsilon=\left(\frac{\mu}{4}+\frac{1}{2}\right)^{2}-\frac{s^{\prime \prime}(0)}{2}
$$

The desired solution of eq $\mathrm{A} 8$ is

$$
\beta=-2 \phi+\sqrt{3 \phi^{2}+\varepsilon}
$$

To determine $\tau_{1}$, we note that eq 13 implies that

$$
\frac{\mathrm{d}\left(s^{1 / 3}\right)}{\mathrm{d} \tau}=\lambda+\frac{1}{3}\left(\frac{1}{c_{\mathrm{s}}}-1\right)-\frac{\lambda \tau_{1}}{3}=a_{0}-a_{1} \tau_{1}
$$

when $s=0$, i.e., when $\tau=\tau_{1}$. The second equality defines the two constants

$$
a_{0}=\lambda+\frac{1}{3}\left(\frac{1}{c_{s}}-1\right)
$$

and

$$
a_{1}=\frac{\lambda}{3}
$$

From eq 24, we then obtain

$$
\frac{\mathrm{d}\left(\tilde{s}^{1 / 3}\right)}{\mathrm{d} \tau}=-\frac{\left(A \mathrm{e}^{-\mu \tau_{1}}+B\right)^{1 / 3}}{\tau_{1}(1-a)^{2 / 3}}
$$

(notice that $\tilde{\tilde{s}}^{1 / 3}$ is the product of three factors and the only nonzero result is obtained when $1-\tau / \tau_{1}$ is differentiated). We find by a combination of eqs A12 and A15 that

$$
\left(A \mathrm{e}^{-\mu / \omega}+B\right) \omega^{8}+\beta^{2}\left(a_{0} \omega-a_{1}\right)^{3}=0
$$

where the aforementioned substitutions $a=1-b, \beta=b / \tau_{1}$ and $\omega=1 / \tau_{1}$ have been made. It is generally a good approximation to neglect $A \mathrm{e}^{-\mu \tau_{1}}=A \mathrm{e}^{-\mu / \omega}$ in comparison with $B$ since the former represents the initial transient. However, this term has nevertheless been retained above since an iterative solution of eq A16 is required. To this end, we may note that eq 12 implies that

$$
\frac{1}{3+1 /\left(\lambda c_{\mathrm{s}}\right)} \leq \omega \leq \frac{1}{3}
$$

since $u$ must attain a value between 0 and 1 .

\section{ASSOCIATED CONTENT}

\section{SI Supporting Information}

The Supporting Information is available free of charge at https://pubs.acs.org/doi/10.1021/acs.molpharmaceut.0c00163.

Visual Basic (VBA) implementation of functions used to perform model calculations in, e.g., Microsoft Excel (PDF)

\section{AUTHOR INFORMATION}

\section{Corresponding Author}

Göran Frenning - Department of Pharmacy and the Swedish Drug Delivery Center (SweDeliver), Uppsala University, 75123 Uppsala, Sweden; 이 orcid.org/0000-0003-4013-9704; Phone: +46 18471 4375; Email: goran.frenning@ farmaci.uu.se; Fax: +4618 4714223 


\section{Authors}

Irès van der Zwaan - Department of Pharmacy and the Swedish Drug Delivery Center (SweDeliver), Uppsala University, 75123 Uppsala, Sweden

Frans Franek - Inhaled Product Development, Pharmaceutical Technology \& Development, AstraZeneca, 43183 Gothenburg, Sweden; 이이.org/0000-0001-5605-4655

Rebecca Fransson - Advanced Drug Delivery, Pharmaceutical Sciences, R\&D, AstraZeneca, 43183 Gothenburg, Sweden

Ulrika Tehler - Advanced Drug Delivery, Pharmaceutical Sciences, R\&D, AstraZeneca, 43183 Gothenburg, Sweden

Complete contact information is available at:

https://pubs.acs.org/10.1021/acs.molpharmaceut.0c00163

\section{Notes}

The authors declare no competing financial interest.

\section{ACKNOWLEDGMENTS}

This study is part of the science program of the Swedish Drug Delivery Center (SweDeliver), and financial support from Vinnova (Dnr 2017-02690) is gratefully acknowledged.

\section{REFERENCES}

(1) Velaga, S. P.; Djuris, J.; Cvijic, S.; Rozou, S.; Russo, P.; Colombo, G.; Rossi, A. Dry powder inhalers: An overview of the in vitro dissolution methodologies and their correlation with the biopharmaceutical aspects of the drug products. Eur. J. Pharm. Sci. 2018, 113, $18-28$.

(2) Radivojev, S.; Zellnitz, S.; Paudel, A.; Fröhlich, E. Searching for physiologically relevant in vitro dissolution techniques for orally inhaled drugs. Int. J. Pharm. 2019, 556, 45-56.

(3) Davies, N. M.; Feddah, M. I. R. A novel method for assessing dissolution of aerosol inhaler products. Int. J. Pharm. 2003, 255, 175187.

(4) Jaspart, S.; Bertholet, P.; Piel, G.; Dogne, J.-M.; Delattre, L.; Evrard, B. Solid lipid microparticles as a sustained release system for pulmonary drug delivery. Eur. J. Pharm. Biopharm. 2007, 65, 47-56.

(5) Cook, R. O.; Pannu, R. K.; Kellaway, I. W. Novel sustained release microspheres for pulmonary drug delivery. J. Controlled Release 2005, 104, 79-90.

(6) Franz, T. J. Percutaneous absorption - relevance of in vitro data. J. Invest. Dermatol. 1975, 64, 190-195.

(7) Salama, R. O.; Traini, D.; Chan, H.-K.; Young, P. M. Preparation and characterisation of controlled release co-spray dried drug-polymer microparticles for inhalation 2: Evaluation of in vitro release profiling methodologies for controlled release respiratory aerosols. Eur. J. Pharm. Biopharm. 2008, 70, 145-152.

(8) Arora, D.; Shah, K. A.; Halquist, M. S.; Sakagami, M. In Vitro Aqueous Fluid-Capacity-Limited Dissolution Testing of Respirable Aerosol Drug Particles Generated from Inhaler Products. Pharm. Res. 2010, 27, 786-795.

(9) Franek, F.; Fransson, R.; Thörn, H.; Bäckman, P.; Andersson, P. U.; Tehler, U. Ranking in Vitro Dissolution of Inhaled Micronized Drug Powders including a Candidate Drug with Two Different Particle Sizes. Mol. Pharmaceutics 2018, 15, 5319-5326.

(10) Gerde, P.; Malmlöf, M.; Havsborn, L.; Sjöberg, C.-O.; Ewing, P.; Eirefelt, S.; Ekelund, K. DissolvIt: An In Vitro Method for Simulating the Dissolution and Absorption of Inhaled Dry Powder Drugs in the Lungs. Assay Drug Dev. Technol. 2017, 15, 77-88.

(11) Eedara, B. B.; Tucker, I. G.; Das, S. C. In vitro dissolution testing of respirable size anti-tubercular drug particles using a small volume dissolution apparatus. Int. J. Pharm. 2019, 559, 235-244.

(12) Son, Y.-J.; McConville, J. T. Development of a standardized dissolution test method for inhaled pharmaceutical formulations. Int. J. Pharm. 2009, 382, 15-22.
(13) Franek, F.; Nilsson, L.; Thörn, H.; Fransson, R.; Tehler, U. Ranking in vitro dissolution of orally inhaled drug substance powders in a time-efficient manner. J. Aerosol Med. Pulm. Drug Delivery 2019, 32, A17.

(14) May, S.; Jensen, B.; Weiler, C.; Wolkenhauer, M.; Schneider, M.; Lehr, C.-M. Dissolution Testing of Powders for Inhalation: Influence of Particle Deposition and Modeling of Dissolution Profiles. Pharm. Res. 2014, 31, 3211-3224.

(15) Noyes, A. A.; Whitney, W. R. The rate of solution of solid substances in their own solutions. J. Am. Chem. Soc. 1897, 19, 930934.

(16) Nernst, W. Theorie der Reaktionsgeschwindigkeit in heterogenen Systemen. Z. Phys. Chem 1904, 47U, 52.

(17) Brunner, E. Reaktionsgeschwindigkeit in heterogenen Systemen. Z. Phys. Chem. 1904, 47U, 56.

(18) Fick, A. Ueber Diffusion. Ann. Phys. Chem. 1855, 170, 59-86.

(19) Siepmann, J.; Siepmann, F. Mathematical modeling of drug dissolution. Int. J. Pharm. 2013, 453, 12-24.

(20) Hixson, A. W.; Crowell, J. H. Dependence of reaction velocity upon surface and agitation I - Theoretical consideration. Ind. Eng. Chem. 1931, 23, 923-931.

(21) Frenning, G.; Strömme, M. Drug release modeled by dissolution, diffusion, and immobilization. Int. J. Pharm. 2003, 250, $137-145$.

(22) Sugano, K. Theoretical comparison of hydrodynamic diffusion layer models used for dissolution simulation in drug discovery and development. Int. J. Pharm. 2008, 363, 73-77.

(23) Frenning, G.; Brohede, U.; Strömme, M. Finite element analysis of the release of slowly dissolving drugs from cylindrical matrix systems. J. Controlled Release 2005, 107, 320-329.

(24) Abramowitz, M.; Stegun, I. A. Handbook of Mathematical Functions; Dover Publications, Inc: New York, 1965.

(25) Paciorek, K. A. Algorithm 385: Exponential integral Ei(x). Commun. ACM 1970, 13, 446-447.

(26) Sakagami, M.; Li, H.; Venitz, J. In Vivo-Relevant Transwell Dish-Based Dissolution Testing for Orally Inhaled Corticosteroid Products. Pharm. Res. 2019, 36, No. 95.

(27) Higuchi, T. Rate of release of medicaments from ointment bases containing drugs in suspension. J. Pharm. Sci. 1961, 50, 874875 .

(28) Higuchi, T. Mechanism of sustained-action medication Theoretical analysis of rate of release of solid drugs dispersed in solid matrices. J. Pharm. Sci. 1963, 52, 1145-1149.

(29) Youdim, K. A.; Avdeef, A.; Abbott, N. J. In vitro transmonolayer permeability calculations: often forgotten assumptions. Drug Discovery Today 2003, 8, 997-1003.

(30) Frenning, G. Modelling drug release from inert matrix systems: From moving-boundary to continuous-field descriptions. Int. J. Pharm. 2011, 418, 88-99.

(31) Rohrschneider, M.; Bhagwat, S.; Krampe, R.; Michler, V.; Breitkreutz, J.; Hochhaus, G. Evaluation of the Transwell System for Characterization of Dissolution Behavior of Inhalation Drugs: Effects of Membrane and Surfactant. Mol. Pharmaceutics 2015, 12, 26182624. 